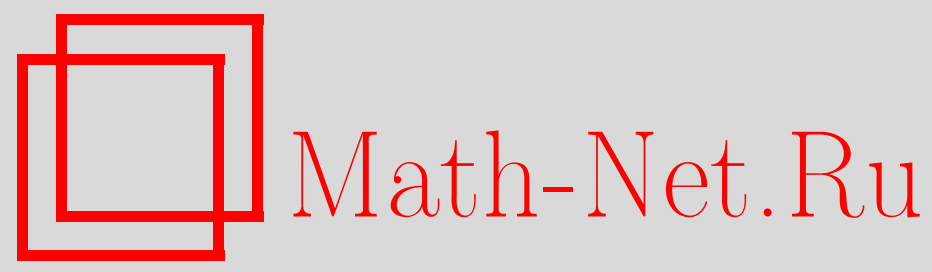

T. Е. Панов, Классификация с точностью до кобордизма многообразий, несущих простое действие группы $\mathbb{Z} / p, M a$ тем. заметки, 1998, том 63, выпуск 2, 260-268

DOI: https://doi.org/10.4213/mzm1272

Использование Общероссийского математического портала Math-Net.Ru подразумевает, что вы прочитали и согласны с пользовательским соглашением http://www . mathnet.ru/rus/agreement

Параметры загрузки:

IP : 3.93 .64 .190

26 апреля 2023 г., 16:23:27

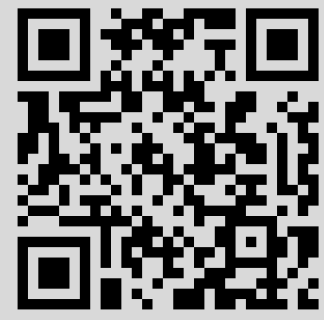




\section{КЛАССИФИКАЦИЯ С ТОЧНОСТЬЮ ДО КОБОРДИЗМА МНОГООБРАЗИЙ, НЕСУЩИХ ПРОСТОЕ ДЕЙСТВИЕ ГРУППЫ $\mathbb{Z} / p$}

\section{Т. Е. Панов}

Получено полное описание множества классов кобордизма квазикомплексных многообразий с действием группы $\mathbb{Z} / p$, множество неподвижных точек которого имеет тривиальное нормальное расслоение, в терминах коэффициентов формальной группы геометрических кобордизмов и в терминах характеристических чисел. Установлена связь с другими работами в данном направлении, где рассматриваемая проблема была решена в различных частных случаях.

Библиография: 7 названий.

ОПРЕдЕлЕниЕ 1 . Назовем действие группы $\mathbb{Z} / p$ на квазикомплексном многообразии $M^{2 n}$ nростым, если множество неподвижных точек представляет собой объединение конечного числа подмногообразий, имеюших тривиальное нормальное расслоение, и сильно простым, если кроме того наборы весов (собственных чисел дифференциала отображения, соответствующего образующей $\rho \in \mathbb{Z} / p$, в неподвижных точках) одинаковы для всех неподвижных подмногообразий одной размерности.

В работе получена полная классификация классов кобордизма $\sigma \in \Omega_{U}$, в которых содержатся многообразия, несущие простое действие групы $\mathbb{Z} / p$ в терминах коэффициентов формальной групш геометрических кобордизмов (теорема 1 ) и в терминах характеристических чисел (теорема 2 , следствие 2 ).

Для сильно простых действий $\mathbb{Z} / p$ проблема классификации была полностью решена Коннером и Флойдом в работе [1] (сильно простое действие в смысле определения 1 назьвалось в [1] действием $\mathbb{Z} / p$, множество неподвижных точек которого имеет тривиальное нормальное расслоение). Обратим внимание на то, что даже в случае действия с изолированными неподвижными точками понятия простого и сильно простого действия отличаются (соответствуюшие примеры приведены в работе). Результаты Коннера и Флойда получены в качестве следствия в данной работе. В то же время методы, используемые в [1], судя по всему, не позволяют получить наш, более общий, результат.

Начиная с работы [2], для решения задач, связанных с действиями $\mathbb{Z} / p$, использовалась теория формальных групп, которая вошла в топологию благодаря формальной группе геометрических кобордизмов. Рассматриваемая проблема впервые четко сформулирована в работе [3], где была получена формула, выражающая класс кобордизма $\bmod p$ многообразия $M^{2 n}$ с простым действием $\mathbb{Z} / p$ через инварианты действия (см.

Работа выполнена при финансовой поддержке Российского фонда фундаментальных исследований, грант № 96-01-01404. 
формулу (7)). Фактически, первые результаты по этой проблеме получены в работе [4] и, в частности, результат, сформулированный у нас как следствие 3 . В [4], как и в этой работе, используется описание множества классов кобордизмов, представленных многообразиями с простым действием $\mathbb{Z} / p$ как $\Omega_{U}$-модуля, натянутого на некоторую систему коэффициентов степенной системы, определяемой формальной группой геометрических кобордизмов. Новый набор образуюших этого $\Omega_{U}$-модуля, введенный нами, позволил решить классификационную задачу в терминах характеристических чисел.

Итак, пусть на квазикомплексном многообразии $M^{2 n}$ действует преобразование $g$ простого периода $p>2$ (т.е. $g^{p}=\mathrm{id}$ ), так что множество неподвижных точек представляет собой объединение подмногообразий с тривиальными нормальными расслоениями (например, лишь изолированные неподвижные точки), т.е. задано простое $\mathbb{Z} / p$-действие. Пусть множество неподвижных точек преобразования $g$ представляет собой объединение неподвижных подмногообразий классов $\lambda_{j} \in \Omega_{U}$, имеюших веса $\left(x_{k}^{(j)}\right) \in(\mathbb{Z} / p)^{*}$ (т.е. неединичные собственные числа дифференциала отображения $g$ в неподвижных точках) в тривиальных нормальных расслоениях к ним. Эти данные определяют класс кобордизма многообразия $M^{2 n}$ в $\Omega_{U}$ с точностью до элементов из $p \Omega_{U}$ (см. [3]), так как в любом классе кобордизмов из $p \Omega_{U}$, очевидно, имеется многообразие, допускающее действие $\mathbb{Z} / p$ (переставляющее компоненты связности) без неподвижных точек.

Каждому неподвижному подмногообразию класса $\lambda_{j} \in \Omega_{U}$ с весами $\left(x_{1}^{(j)}, \ldots, x_{m_{j}}^{(j)}\right)$, $2 m_{j}+\operatorname{dim} \lambda_{j}=2 n$, соответствует инвариант Коннера-Флойда

$$
\alpha_{2 m_{j}-1}\left(x_{1}^{(j)}, \ldots, x_{m_{j}}^{(j)}\right) \in U_{2 m_{j}-1}(B \mathbb{Z} / p)
$$

(см. [2]). Кольцо кобордизмов $U^{*}(B \mathbb{Z} / p)$ имеет вид $U^{*}(B \mathbb{Z} / p)=\Omega_{U}[[u]] /[u]_{p}=0$, где $[u]_{p}=p \Psi^{p}(u)-p$-я степень в формальной групе геометрических кобордизмов (см. [3]). Из того что $D\left(\alpha_{i}(1, \ldots, 1)\right)=u^{n-i}$, где $D$ - оператор двойственности Пуанкаре-Атья

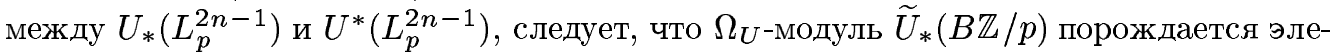
ментами $\alpha_{2 i-1}(1, \ldots, 1)$ и соотношениями

$$
0=\frac{[u]_{p}}{u} \cap \alpha_{2 i-1}(1, \ldots, 1),
$$

где "П" - оператор "высечения" Чеха, $u^{k} \cap \alpha_{2 i-1}(1, \ldots, 1)=\alpha_{2(i-k)-1}(1, \ldots, 1)$.

В [5] было доказано, что

$$
\alpha_{2 k-1}\left(x_{1}, \ldots, x_{k}\right)=\left(\prod_{j=1}^{k} \frac{u}{[u]_{x_{j}}}\right) \cap \alpha_{2 k-1}(1, \ldots, 1) .
$$

Расширив систему образуюших модуля $\widetilde{U}_{*}(B \mathbb{Z} / p)$ элементами $\alpha_{2 k-1}\left(x_{1}, \ldots, x_{k}\right)$ для $x_{j} \neq 1 \bmod p$ и добавив к соотношениям $(1)$ соотношения $(2)$, получим $\Omega_{U} \otimes \mathbb{Z}_{(p)}$-свободную резольвенту модуля $\widetilde{U}_{*}(B \mathbb{Z} / p)$ (здесь $\mathbb{Z}_{(p)}$ - кольцо рациональньх чисел, знаменатели которых взаимно просты с $p$, которое можно рассматривать как кольцо $p$-адических чисел):

$$
0 \longrightarrow F_{1} \longrightarrow F_{0} \longrightarrow \widetilde{U}_{*}(B \mathbb{Z} / p) \longrightarrow 0,
$$

где $F_{0}$ - свободный $\Omega_{U} \otimes \mathbb{Z}_{(p)}$-модуль , натянутьй на образуюшие $\alpha_{2 k-1}\left(x_{1}, \ldots, x_{k}\right), F_{1}-$

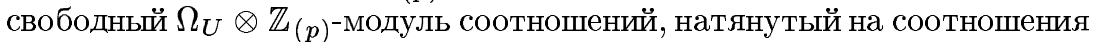

$$
\begin{gathered}
a\left(x_{1}, \ldots, x_{k}\right)=\alpha_{2 k-1}\left(x_{1}, \ldots, x_{k}\right)-\left(\prod_{j=1}^{k} \frac{u}{[u]_{x_{j}}}\right) \cap \alpha_{2 k-1}(1, \ldots, 1), \\
a_{k}=\frac{[u]_{p}}{u} \cap \alpha_{2 k-1}(1, \ldots, 1) .
\end{gathered}
$$


Каждое простое действие $\mathbb{Z} / p$ на $M^{2 n}$ задает соотношение между элементами $\alpha_{2 k-1}\left(x_{1}, \ldots, x_{k}\right)$ в $\widetilde{U}_{*}(B \mathbb{Z} / p)$. В силу определения элементов $\widetilde{U}_{*}(B \mathbb{Z} / p)$ как классов бордизмов многообразий со свободным действием групшы $\mathbb{Z} / p$ верно и обратное: каждое соотношение в $\widetilde{U}_{*}(B \mathbb{Z} / p)$ вида

$$
\sum_{j} \lambda_{j} \alpha_{2 m_{j}-1}\left(x_{1}^{(j)}, \ldots, x_{m_{j}}^{(j)}\right)=0, \quad \lambda_{j} \in \Omega_{U}, 2 m_{j}+\operatorname{dim} \lambda_{j}=2 n
$$

реализуется на некотором многообразии $M^{2 n}$ с простым действием $\mathbb{Z} / p\left(M^{2 n}\right.$ получается из “пленки" со свободным действием $\mathbb{Z} / p$, натянутой на объединение многообразий $\lambda_{j} \times S^{2 m_{j}-1}$, соответствующей соотношению в $\widetilde{U}_{*}(B \mathbb{Z} / p)$, путем приклеивания $\left.\lambda_{j} \times D^{2 m_{j}}\right)$, причем класс кобордизма $M^{2 n}$ в $\Omega_{U}$ определен однозначно с точностью до элементов $p \Omega_{U}$. Таким образом, определен гомоморфизм реализации

$$
\Phi: F_{1} \rightarrow \Omega_{U} / p \Omega_{U}=\Omega_{U} \otimes \mathbb{Z} / p
$$

ставяший в соответствие соотношению между $\alpha_{2 k-1}\left(x_{1}, \ldots, x_{k}\right) \in \widetilde{U}_{*}(B \mathbb{Z} / p)$ класс кобордизмов $\bmod p$ многообразия, на котором это соотношение реализуется в указанном выше смысле. В силу результатов Коннера и Флойда (см. [1], а также [4]) для базисньх соотношений из $F_{1}$ имеем

$$
\begin{gathered}
\Phi\left(a\left(x_{1}, \ldots, x_{k}\right)\right)=\left\langle\prod_{i=1}^{k} \frac{u}{[u]_{x_{i}}}\right\rangle_{k} \bmod p \in \Omega_{U} / p \Omega_{U} \\
\Phi\left(a_{k}\right)=-\left\langle\frac{[u]_{p}}{u}\right\rangle_{k} \bmod p \in \Omega_{U} / p \Omega_{U}
\end{gathered}
$$

где $\langle\cdot\rangle_{k}$ означает коэффициент при $u^{k}$. Отсюда следует, что $\operatorname{Im} \Phi=\widetilde{\Lambda}(1) \otimes \mathbb{Z} / p$, где $\widetilde{\Lambda}(1)=\Lambda^{+}(1) \cdot \Omega_{U}$ есть $\Omega_{U}$-модуль, натянутый на положительную часть $\Lambda^{+}(1)$ кольца коэффициентов $\Lambda(1)$ степенной системы $[u]_{k}$. Гомоморфизм $\Phi$ поднимается до гомоморфизмов $\Phi: F_{1} \rightarrow \widetilde{\Lambda}(1) \otimes \mathbb{Z}_{(p)}$ или $\Phi: F_{1} \rightarrow \widetilde{\Lambda}_{p}(1) \otimes \mathbb{Z}_{(p)}$, где $\widetilde{\Lambda}_{p}(1)$ есть $\Omega_{U \text {-модуль, }}$ натянутьй на $\Lambda^{+}(1)$ и $p$.

Таким образом, задача об описании множества классов кобордизмов многообразий с простым действием $\mathbb{Z} / p$ сводится к описанию $\Omega_{U} \otimes \mathbb{Z} / p$-модуля $\widetilde{\Lambda}(1) \otimes \mathbb{Z} / p$ или $\Omega_{U} \otimes \mathbb{Z}_{(p)}$-модулей $\widetilde{\Lambda}(1) \otimes \mathbb{Z}_{(p)}$ и $\widetilde{\Lambda}_{p}(1) \otimes \mathbb{Z}_{(p)}$, которые также являются идеалами в $\Omega_{U} \otimes \mathbb{Z} / p$ и $\Omega_{U} \otimes \mathbb{Z}_{(p)}$ соответственно.

Пусть

$$
[u]_{k}=k u+\sum_{n \geqslant 1} \alpha_{n}^{(k)} u^{n+1}
$$

$\alpha_{n}^{(k)} \in \Omega_{U}^{-2 n}-$ коэффициенты степенной системы.

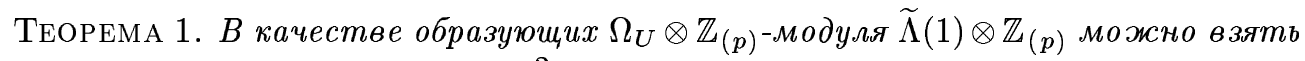
следующие коэффициенты $\alpha_{n} \in \Omega_{U}^{-2 n}$ :

$$
\alpha_{n}=\left\{\begin{array}{ll}
\alpha_{n}^{\left(p_{1}\right)}, & \text { если } n \text { не делится на } p-1, \\
\alpha_{n}^{(p)}, & \text { если } п \text { делится на } p-1,
\end{array} \quad n=1,2, \ldots,\right.
$$

где $p_{1}-$ простой первообразный корень $\bmod p\left(\right.$ m.е. $p_{1}-$ образующая чиклической группь $\left.(\mathbb{Z} / p)^{*}\right)$. 
ЗАмЕчАнИЕ. Из теоремы Дирихле следует, что среди всех первообразных корней $\bmod p$ имеются простые числа.

ДокАЗАТЕЛЬСтво ТЕОРЕмы 1. Рассмотрим вначале коэффициенты $\alpha_{n}^{(r)}$, где $r$ не является простьм. Пусть $r=p_{1} q$, где $p_{1}$ - простое число. Так как $[x]_{r}=\left[[x]_{p_{1}}\right]_{q}$, имеем

$$
\begin{aligned}
r x+\sum_{n} \alpha_{n}^{(r)} x^{n+1} & =q[x]_{p_{1}}+\sum_{n} \alpha_{n}^{(q)}\left([x]_{p_{1}}\right)^{n+1} \\
& =p_{1} q x+q \sum_{n} \alpha_{n}^{\left(p_{1}\right)} x^{n+1}+\sum_{n} \alpha_{n}^{(q)}\left(p_{1} x+\sum_{m} \alpha_{m}^{\left(p_{1}\right)} x^{m+1}\right)^{n+1} .
\end{aligned}
$$

Приравнивая коэффищиенты при одинаковых степенях $x$, получим $\alpha_{n}^{(r)}=P\left(\alpha_{1}^{\left(p_{1}\right)}, \ldots\right.$, $\left.\alpha_{n}^{\left(p_{1}\right)}, \alpha_{1}^{(q)}, \ldots, \alpha_{n}^{(q)}\right)$, где $P$ - некоторый полином с целыми коэффициентами (без свободного члена). Отсюда получаем представление

$$
\alpha_{n}^{(r)}=\lambda_{1} \alpha_{1}^{\left(p_{1}\right)}+\cdots+\lambda_{n} \alpha_{n}^{\left(p_{1}\right)}+\mu_{1} \alpha_{1}^{(q)}+\cdots+\mu_{n} \alpha_{n}^{(q)}, \quad \lambda_{i}, \mu_{i} \in \Omega_{U} .
$$

Таким образом, коэффициенты $\alpha_{n}^{(r)}, r=p_{1} q$, можно исключить из системы образующих $\Omega_{U} \otimes \mathbb{Z}_{(p)}$-модуля $\widetilde{\Lambda}(1) \otimes \mathbb{Z}_{(p)}$. Если $q$ снова не является простым, то повторяем эту процедуру, и т.д. В итоге получаем систему образующих модуля $\widetilde{\Lambda}(1) \otimes \mathbb{Z}_{(p)}$, состоящую лишь из коэффициентов $\alpha_{n}^{\left(p_{1}\right)}$ с простьми $p_{1}$. Среди всех простых $p_{1}$ имеется выделенное простое число $p$. Далее покажем, что эту систему образуюших можно сузить до системы (3).

Вначалезаметим, что в размерности 1 (т.е. в $\Omega_{U}^{-2}$ ) в качестве образующей $\widetilde{\Lambda}(1) \otimes \mathbb{Z}_{(p)}$ можно взять $\alpha_{1}^{\left(p_{1}\right)}$, где $p_{1}-$ простой первообразньй корень $\bmod p$. Действительно, пусть $p_{2}-$ любое простое число. Тогда $\left[[x]_{p_{2}}\right]_{p_{1}}=\left[[x]_{p_{1}}\right]_{p_{2}}$, т.е.

$$
\begin{aligned}
& p_{1} p_{2} x+p_{1} \sum_{n} \alpha_{n}^{\left(p_{2}\right)} x^{n+1}+\sum_{n} \alpha_{n}^{\left(p_{1}\right)}\left(p_{2} x+\sum_{m} \alpha_{m}^{\left(p_{2}\right)} x^{m+1}\right)^{n+1} \\
& =p_{2} p_{1} x+p_{2} \sum_{n} \alpha_{n}^{\left(p_{1}\right)} x^{n+1}+\sum_{n} \alpha_{n}^{\left(p_{2}\right)}\left(p_{1} x+\sum_{m} \alpha_{m}^{\left(p_{1}\right)} x^{m+1}\right)^{n+1} .
\end{aligned}
$$

Приравнивая коэффициенты при $x^{2}$, получим $p_{1} \alpha_{1}^{\left(p_{2}\right)}+p_{2}^{2} \alpha_{1}^{\left(p_{1}\right)}=p_{2} \alpha_{1}^{\left(p_{1}\right)}+p_{1}^{2} \alpha_{1}^{\left(p_{2}\right)}$, т.е. $\left(p_{1}-p_{1}^{2}\right) \alpha_{1}^{\left(p_{2}\right)}=\left(p_{2}-p_{2}^{2}\right) \alpha_{1}^{\left(p_{1}\right)}$. Так как $p_{1}-$ первообразньй корень, $p_{1}-p_{1}^{2}$ обратимо в $\mathbb{Z}_{(p)}$. Значит, $\alpha_{1}^{\left(p_{2}\right)}=\lambda \alpha_{1}^{\left(p_{1}\right)}$, где $\lambda \in \mathbb{Z}_{(p)} \subset \Omega_{U} \otimes \mathbb{Z}_{(p)}$, т.е. для любого $p_{2}$ коэффициент $\alpha_{1}^{\left(p_{2}\right)}$ выражается через $\alpha_{1}^{\left(p_{1}\right)}$.

Допустим, мы доказали, что система (3) коэффициентов $\alpha_{1}, \ldots, \alpha_{k}, \ldots$ (т.е. $\alpha_{i}$ - коэффициент при $x^{i+1}$ в $[x]_{p_{1}}$, если $i$ не делится на $p-1$, и коэффициент при $x^{i+1}$ в $[x]_{p}$, если $i$ делится на $p-1)$ является системой образуюших $\widetilde{\Lambda}(1) \otimes \mathbb{Z}_{(p)}$ до размерности $n-1$, т.е. при любом $k \leqslant n-1$ и любом $q$ имеется представление $\alpha_{k}^{(q)}=\lambda_{1}^{(q)} \alpha_{1}+\cdots+\lambda_{k}^{(q)} \alpha_{k}$, $\lambda_{i}^{(q)} \in \Omega_{U} \otimes \mathbb{Z}_{(p)}$. Докажем, что такое представление имеется и для $\alpha_{n}^{(q)}$. Из сказанного выше следует, что достаточно ограничиться простыми $q$. Пусть вначале $n$ не делится на $p-1$, т.е. $\alpha_{n}=\alpha_{n}^{\left(p_{1}\right)}$, где $p_{1}-$ первообразный корень $\bmod p$, и пусть $p_{2}-$ произвольное простое число. Приравняем в (4) коэффициенты при $x^{n+1}$ :

$p_{1} \alpha_{n}^{\left(p_{2}\right)}+p_{2}^{n+1} \alpha_{n}^{\left(p_{1}\right)}+\mu_{1} \alpha_{1}+\cdots+\mu_{n-1} \alpha_{n-1}=p_{2} \alpha_{n}^{\left(p_{1}\right)}+p_{1}^{n+1} \alpha_{n}^{\left(p_{2}\right)}+\nu_{1} \alpha_{1}+\cdots+\nu_{n-1} \alpha_{n-1}$ 
(мы разложили все коэффициенты $\alpha_{k}^{\left(p_{1}\right)}, \alpha_{k}^{\left(p_{2}\right)}, k<n$, по образуюшим $\alpha_{1}, \ldots, \alpha_{n-1}$, т.е. $\left.\mu_{i}, \nu_{i} \in \Omega_{U} \otimes \mathbb{Z}_{(p)}\right)$. Отсюда

$$
p_{1}\left(1-p_{1}^{n}\right) \alpha_{n}^{\left(p_{2}\right)}=\left(p_{2}-p_{2}^{n+1}\right) \alpha_{n}^{\left(p_{1}\right)}+\left(\nu_{1}-\mu_{1}\right) \alpha_{1}+\cdots+\left(\nu_{n-1}-\mu_{n-1}\right) \alpha_{n-1} .
$$

Так как $p_{1}-$ первообразный корень $\bmod p$ и $n$ не делится на $p-1$, то $p_{1}\left(1-p_{1}^{n}\right)$ обратимо в $\mathbb{Z}_{(p)}$. Тогда из (5) получаем выражение $\alpha_{n}^{\left(p_{2}\right)}$ через $\alpha_{1}, \ldots, \alpha_{n-1}$ и $\alpha_{n}=\alpha_{n}^{\left(p_{1}\right)} \mathrm{c}$ коэффициентами в $\Omega_{U} \otimes \mathbb{Z}_{(p)}$.

Пусть теперь $n$ делится на $p-1$. Сделаем вначале несколько предварительных замечаний. Известно, что $\Omega_{U}$ является кольцом полиномов: $\Omega_{U}=\mathbb{Z}\left[a_{1}, a_{2}, \ldots, a_{n}, \ldots\right]$, $a_{n} \in \Omega_{U}^{-2 n}$. Кольцо $\Omega_{U}$ является кольцом коэффициентов (универсальной) формальной группы геометрических кобордизмов, а кольцом коэффициентов логарифма этой формальной групшы является кольцо $\Omega_{U}(\mathbb{Z})=\mathbb{Z}\left[b_{1}, b_{2}, \ldots, b_{n}, \ldots\right]$,

$$
b_{n}=\frac{\mathbb{C P}_{n}}{n+1}, \quad g(u)=u+\sum_{n} \frac{\mathbb{C P}_{n}}{n+1} u^{n+1} .
$$

При этом в кольцах $\Omega_{U}, \Omega_{U}(\mathbb{Z})$ можно выбрать мультипликативные образующие $\left\{a_{i}^{*}\right\}$, $\left\{b_{i}^{*}\right\}$ так, что вложение $\iota_{0}: \Omega_{U} \rightarrow \Omega_{U}(\mathbb{Z})$ задается следуюшим образом:

$$
\iota_{0}\left(a_{i}^{*}\right)= \begin{cases}p \cdot b_{i}^{*}, & \text { если } i=p^{k}-1 \text { для некоторого } k, \\ b_{i}^{*} & \text { в остальных случаях. }\end{cases}
$$

Пусть $B^{+}$- положительная часть кольца $B=\Omega_{U}(\mathbb{Z})$. Тогда $\left(B^{+}\right)^{2}$ - разложимые элементы в $\Omega_{U}(\mathbb{Z})$. При вложении $\iota_{0}: \Omega_{U} \rightarrow \Omega_{U}(\mathbb{Z})$ коэффициенты $\alpha_{n}^{(p)}$ ряда $[x]_{p}$ переходят в элементы $\left(p-p^{n+1}\right) b_{n}+\left(B^{+}\right)^{2}$. Таким образом, коэффициенты $\alpha_{p^{k}-1}^{(p)}$ можно взять за мультипликативные образуюшие кольца $\Omega_{U} \otimes \mathbb{Z}_{(p)}$ в размерностях $p^{k}-1$. В размерностях $l \neq p^{k}-1$ имеем $\alpha_{l}^{(p)} \in p \Omega_{U}$, т.е. $\alpha_{l}^{(p)}$ делится на $p$ в $\Omega_{U}$.

Перепишем соотношение (4), подставляя $p$ вместо $p_{1}$ :

$$
\begin{aligned}
p p_{2} x & +p \sum_{m} \alpha_{m}^{\left(p_{2}\right)} x^{m+1}+\sum_{m} \alpha_{m}^{(p)}\left(p_{2} x+\alpha_{1}^{\left(p_{2}\right)} x^{2}+\alpha_{2}^{\left(p_{2}\right)} x^{3}+\cdots\right)^{m+1} \\
= & p_{2} p x+p_{2} \sum_{m} \alpha_{m}^{(p)} x^{m+1}+\sum_{m} \alpha_{m}^{\left(p_{2}\right)}\left(p x+\alpha_{1}^{(p)} x^{2}+\alpha_{2}^{(p)} x^{3}+\cdots\right)^{m+1} .
\end{aligned}
$$

Приравняем коэффициенты при $x^{n+1}$ :

$$
\begin{gathered}
p \alpha_{n}^{\left(p_{2}\right)}+p_{2}^{n+1} \alpha_{n}^{(p)}+\left\langle\sum_{m<n} \alpha_{m}^{(p)}\left(p_{2} x+\alpha_{1}^{\left(p_{2}\right)} x^{2}+\alpha_{2}^{\left(p_{2}\right)} x^{3}+\cdots\right)^{m+1}\right\rangle_{n+1} \\
=p_{2} \alpha_{n}^{(p)}+p^{n+1} \alpha_{n}^{\left(p_{2}\right)}+\left\langle\sum_{m<n} \alpha_{m}^{\left(p_{2}\right)}\left(p x+\alpha_{1}^{(p)} x^{2}+\alpha_{2}^{(p)} x^{3}+\cdots\right)^{m+1}\right\rangle_{n+1},
\end{gathered}
$$

где $\langle\cdot\rangle_{n+1}$ означает коэффициент при $x^{n+1}$. Разложим коэффициенты $\alpha_{m}^{\left(p_{2}\right)}$ при $m<n$ по образующим $\alpha_{1}, \ldots, \alpha_{m}$. Так как $\alpha_{m}^{(p)} \in p \Omega_{U}$ при $m \neq p^{k}-1$, перепишем последнее соотношение в виде

$$
\begin{aligned}
p\left(1-p^{n}\right) \alpha_{n}^{\left(p_{2}\right)}= & p_{2}\left(1-p_{2}^{n}\right) \alpha_{n}^{(p)}+p\left(\mu_{1} \alpha_{1}+\cdots+\mu_{n} \alpha_{n}\right) \\
& -\left\langle\sum_{k: p^{k}-1<n} \alpha_{p^{k}-1}^{(p)}\left(p_{2} x+\alpha_{1}^{\left(p_{2}\right)} x^{2}+\alpha_{2}^{\left(p_{2}\right)} x^{3}+\cdots\right)^{p^{k}}\right\rangle_{n+1} \\
& +\left\langle\sum_{m<n} \alpha_{m}^{\left(p_{2}\right)}\left(\alpha_{p-1}^{(p)} x^{p}+\alpha_{p^{2}-1}^{(p)} x^{p^{2}}+\cdots+\alpha_{p^{k}-1}^{(p)} x^{p^{k}}+\cdots\right)^{m+1}\right\rangle_{n+1} .
\end{aligned}
$$


Последние два слагаемых мы можем записать в виде $\alpha_{p-1}^{(p)} \nu_{1}+\alpha_{p^{2}-1}^{(p)} \nu_{2}+\cdots+\alpha_{p^{k}-1}^{(p)} \nu_{k}$, где $p^{k}-1<n, \nu_{i} \in \Omega_{U}$. Так как остальная часть соотношения (6) лежит в $p \Omega_{U} \otimes \mathbb{Z}_{(p)}$ (т.е. делится на $p)$, а $\alpha_{p^{i}-1}^{(p)}$ являются полиномиальными образуюшими $\Omega_{U} \otimes \mathbb{Z}_{(p)}$ в размерностях $p^{i}-1$, отсюда следует, что и $\nu_{i} \in p \Omega_{U} \otimes \mathbb{Z}_{(p)}$, т.е. $\nu_{i}$ делятся на $p$ в $\Omega_{U} \otimes \mathbb{Z}_{(p)}$. Пусть $\nu_{i}=p \varkappa_{i}, \varkappa_{i} \in \Omega_{U} \otimes \mathbb{Z}_{(p)}$. Тогда (6) можно записать в виде

$$
\begin{aligned}
& p\left(1-p^{n}\right) \alpha_{n}^{\left(p_{2}\right)}=p_{2}\left(1-p_{2}^{n}\right) \alpha_{n}^{(p)}+p\left(\mu_{1} \alpha_{1}+\cdots+\mu_{n} \alpha_{n}\right) \\
& \quad+p\left(\alpha_{p-1}^{(p)} \varkappa_{1}+\alpha_{p^{2}-1}^{(p)} \varkappa_{2}+\cdots+\alpha_{p^{k}-1}^{(p)} \varkappa_{k}\right), \quad p^{k}-1<n, \quad \mu_{i}, \varkappa_{i} \in \Omega_{U} \otimes \mathbb{Z}_{(p)} .
\end{aligned}
$$

Так как $n$ делится на $p-1$, то $1-p_{2}^{n}$ делится на $p$ (при $p_{2} \neq p$ ). Таким образом, все соотношение делится на $p$. Разделим его на $p$, учитьвая, что $1-p^{n}$ обратимов $\Omega_{U} \otimes \mathbb{Z}_{(p)}$, получим, что $\alpha_{n}^{\left(p_{2}\right)}$ представляется в виде

$$
\alpha_{n}^{\left(p_{2}\right)}=\frac{p_{2}\left(1-p_{2}^{n}\right)}{p\left(1-p^{n}\right)} \alpha_{n}^{(p)}+\lambda_{1} \alpha_{1}+\cdots+\lambda_{n-1} \alpha_{n-1} .
$$

Полагая

$$
\lambda_{n}=\frac{p_{2}\left(1-p_{2}^{n}\right)}{p\left(1-p^{n}\right)} \in \Omega_{U} \otimes \mathbb{Z}_{(p)},
$$

получим искомое представление и для $\alpha_{n}^{\left(p_{2}\right)}$ (так как $\left.\alpha_{n}=\alpha_{n}^{(p)}\right)$. Теорема доказана.

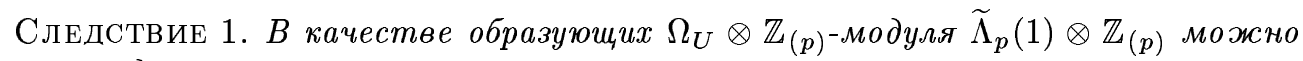
взять следующие:

$$
\alpha_{n}= \begin{cases}p, & \text { если } n=0, \\ \alpha_{n}^{\left(p_{1}\right)}, & \text { если } n \text { не делится на } p-1, p_{1}-\text { первообразный корень } \bmod p \\ \alpha_{p^{k}-1}^{(p)}, & \text { если } n=p^{k}-1, k=1,2, \ldots\end{cases}
$$

В качестве образующих $\Omega_{U} \otimes \mathbb{Z} / p$-модуля $\widetilde{\Lambda}(1) \otimes \mathbb{Z} / p$ можнно взять

$$
\alpha_{n}= \begin{cases}\alpha_{n}^{\left(p_{1}\right)}, & \text { если } n \text { не делится на } p-1, \\ \alpha_{p^{k}-1}^{(p)}, & \text { если } n=p^{k}-1, k=1,2, \ldots\end{cases}
$$

В остальных размерностях образующих нет.

ДокАЗАТЕЛЬСтво. Это следует из того, что для системы образуюших $\widetilde{\Lambda}(1) \otimes \mathbb{Z}_{(p)}$, построенной в теореме 1 при $n$ кратных $p-1$, но $n \neq p^{k}-1$, элементы $\alpha_{n}$ делятся на $p$, т.е. лежат в $p \Omega_{U}$, а остальные $\alpha_{n}$ не лежат в $p \Omega_{U}$.

Далее, используя описание структуры $\Omega_{U} \otimes \mathbb{Z}_{(p)}$-модуля $\widetilde{\Lambda}_{p}(1) \otimes \mathbb{Z}_{(p)}$, докажем результат, аналогичный теореме Стонга-Хаттори, - опишем множество классов кобордизма, имеющих в качестве представителя многообразие с простым действием $\mathbb{Z} / p$ в терминах характеристических чисел.

Как известно из [3], гомоморфизм $\Phi: F_{1} \rightarrow \widetilde{\Lambda}_{p}(1) \otimes \mathbb{Z}_{(p)}$ продолжается до гомоморфизма $\gamma_{p}: F_{0} \rightarrow \Omega_{U}(\mathbb{Z}) \otimes \mathbb{Z}_{(p)}$, причем имеет место формула

$$
\gamma_{p}\left(x_{1}, x_{2}, \ldots, x_{k}\right)=\left\langle\left(\prod_{j=1}^{k} \frac{u}{[u]_{x_{j}}}\right) \frac{p u}{[u]_{p}}\right\rangle_{k}
$$


В частности,

$$
\gamma_{p}(1, \ldots, 1)=\left\langle\frac{p u}{[u]_{p}}\right\rangle_{k}
$$

где $\gamma_{p}\left(x_{1}, x_{2}, \ldots, x_{k}\right):=\gamma_{p}\left(\alpha_{2 k-1}\left(x_{1}, x_{2}, \ldots, x_{k}\right)\right), \alpha_{2 k-1}\left(x_{1}, x_{2}, \ldots, x_{k}\right) \in F_{0}$.

Таким образом, для действия группы $\mathbb{Z} / p$ на $M^{2 n}$ с неподвижными подмногообразиями классов $\lambda_{j} \in \Omega_{U}$, имеюшими веса $\left(x_{k}^{(j)}\right) \in(\mathbb{Z} / p)^{*}$ в тривиальных нормальных расслоениях к ним, имеет место соотношение

$$
\left[M^{2 n}\right] \equiv \sum_{j} \lambda_{j} \gamma_{p}\left(x_{1}^{(j)}, \ldots, x_{m_{j}}^{(j)}\right) \bmod p \Omega_{U}
$$

Возникает вопрос, аналогичный проблеме Милнора-Хирцебруха (ответ на которую дает теорема Стонга-Хаттори), поставленньй в работе [3]: какие из элементов вида

$$
\sum_{j} \lambda_{j} \gamma_{p}\left(x_{1}^{(j)}, \ldots, x_{m_{j}}^{(j)}\right) \in \Omega_{U}(\mathbb{Z}) \otimes \mathbb{Z}_{(p)}
$$

представляют собой классы кобордизма многообразий, несуших простое действие $\mathbb{Z} / p$ ? Ответ на этот вопрос дает теорема 2 .

ОПРЕДЕЛЕНИЕ 2 . Пусть $\omega=\sum_{i} k_{i} \cdot(i), k_{i} \geqslant 0,-$ разбиение числа $n=\|\omega\|=\sum_{i} k_{i} \cdot i$. Назовем разбиение $\omega$ кратным $p-1$, если все $i$, для которых $k_{i} \neq 0$, кратны $p-1$ (естественно, такие разбиения существуют только для $n$, кратных $p-1)$. Назовем разбиение $\omega$ не $p$-адическим, если для любого $j>0 \quad k_{p^{j}-1}=0$.

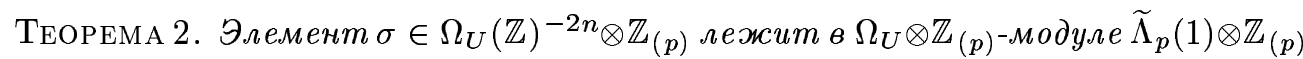
$u$, таким образом, является классом кобордизма многообразия с простым действием $\mathbb{Z} / p$ тогда и только тогда, когда все $K$-характеристические числа $s_{\omega}(\sigma)$,

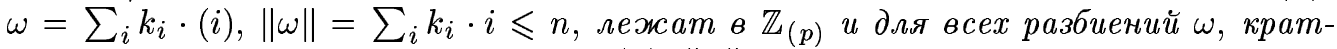
ных $p-1$, когомологические числа $s_{\omega}(\sigma),\|\omega\|=n$, делятся нар.

ДокАЗАТЕЛЬСтвО. Необходимость. Пусть $\sigma \in \widetilde{\Lambda}_{p}(1) \otimes \mathbb{Z}_{(p)}$. Заметим, что система образуюших $\Omega_{U} \otimes \mathbb{Z}_{(p)}$-модуля $\widetilde{\Lambda}_{p}(1) \otimes \mathbb{Z}_{(p)}$, описанная в следствии 1 , обладает тем свойством, что каждый элемент $\alpha_{i} \in \widetilde{\Lambda}_{p}(1) \otimes \mathbb{Z}_{(p)}$ из системы образующих является также полиномиальной образуюшей $\Omega_{U} \otimes \mathbb{Z}_{(p)}$ в размерности $-2 i$. Дополним эту систему образуюших полиномиальными образуюшими $\alpha_{i}$ в недостающих размерностях $-2 i$ таких, что $i$ делится на $p-1$, но $i \neq p^{k}-1$. Таким образом, $\Omega_{U} \otimes \mathbb{Z}_{(p)}=\mathbb{Z}_{(p)}\left[\alpha_{1}, \alpha_{2}, \ldots\right]$. B $\mathbb{Z}_{(p)}$.

Так как $\sigma \in \widetilde{\Lambda}_{p}(1) \otimes \mathbb{Z}_{(p)} \subset \Omega_{U} \otimes \mathbb{Z}_{(p)}$, все $K$-характеристические числа $s_{\omega}(\sigma)$ лежат

Если $n$ не делится на $p-1$, то разбиений $\omega$, кратных $p-1$, не сушествует.

Пусть $n=m(p-1)$. Представим $\sigma$ как полином от $\alpha_{i}$ ( $\sigma$ является однородным полиномом степени $-2 m(p-1))$ :

$$
\sigma=\sum_{\|\omega\|=m(p-1)} r_{\omega} \alpha_{\omega}=r_{m(p-1)} \alpha_{m(p-1)}+\cdots, \quad \omega=\sum_{i} k_{i} \cdot(i), \quad \alpha_{\omega}=\alpha_{1}^{k_{1}} \cdot \alpha_{2}^{k_{2}} \cdots .
$$

Из описания структуры $\widetilde{\Lambda}_{p}(1) \otimes \mathbb{Z}_{(p)}($ см. следствие 1$)$ следует, что $\sigma \in \widetilde{\Lambda}_{p}(1) \otimes \mathbb{Z}_{(p)}$ тогда и только тогда, когда для всех не $p$-адических разбиений $\omega$, кратных $p-1$, коэффициенты $r_{\omega}$ в разложении (8) делятся на $p$. 
Пусть

$$
\operatorname{ch}_{U}(u)=t+\sum_{i \geqslant 1} \beta_{i} t^{i+1}, \quad u=c_{1}^{U}(\zeta) \in U^{2}(\mathbb{C P}(\infty)), \quad t=c_{1}^{H}(\zeta) \in H^{2}(\mathbb{C P}(\infty))
$$

$\operatorname{ch}_{U}$ - характер Чженя-Дольда в кобордизмах (см. [6]), $\beta_{i} \in \Omega_{U}(\mathbb{Z})$. Тогда для любого $\sigma \in \Omega_{U}^{-2 n}$ имеем

$$
\sigma=\sum_{\|\omega\|=n} s_{\omega}(\sigma) \beta_{\omega} \quad \text { и } \quad B=\Omega_{U}(\mathbb{Z})=\mathbb{Z}\left[\beta_{1}, \beta_{2}, \cdots\right] .
$$

Кроме того,

$$
\alpha_{i}= \begin{cases}e_{i} \cdot \beta_{i}+\left(B^{+}\right)^{2} & \text { для } i \neq p^{k}-1, \\ p e_{i} \cdot \beta_{i}+\left(B^{+}\right)^{2} & \text { для } i=p^{k}-1\end{cases}
$$

где $e_{i} \in \mathbb{Z}_{(p)}$ - обратимьй элемент (единица). Таким образом, для всех разбиений $\omega=\sum_{i} k_{i} \cdot(i)$, кратных $p-1$, коэффициент при $\beta_{\omega}$ в разложении $\sigma$ равен

$$
s_{\omega}(\sigma)=\sum_{\omega^{\prime}: \omega^{\prime} \supset \omega} r_{\omega^{\prime}} s_{\omega}\left(\alpha_{\omega^{\prime}}\right)
$$

где $\omega^{\prime} \supset \omega$ означает, что $\omega$ является измельчением разбиения $\omega^{\prime}$. Этот коэффициент делится на $p$. Действительно, если разбиение $\omega^{\prime}=\sum_{i} k_{i}^{\prime} \cdot(i)$ кратно $p-1$ и не $p$-адическое, то $r_{\omega^{\prime}}$ делится на $p$, так как $\sigma \in \widetilde{\Lambda}_{p}(1) \otimes \mathbb{Z}_{(p)}\left(\right.$ см. выше), а если среди $i$ таких, что $k_{i}^{\prime} \neq 0$, есть числа вида $p^{k}-1$, то $\alpha_{\omega^{\prime}} \in p \Omega_{U}(\mathbb{Z}) \otimes \mathbb{Z}_{(p)}$, т.е. $s_{\omega}\left(\alpha_{\omega^{\prime}}\right)$ делится на $p$.

Достаточность. Так как все $K$-характеристические числа $\sigma$ лежат в $\mathbb{Z}_{(p)}$, по теореме Стонга-Хаттори (см. [7]) $\sigma \in \Omega_{U} \otimes \mathbb{Z}_{(p)}$. Пусть, кроме того, для любого разбиения $\omega=\sum_{i} k_{i} \cdot(i),\|\omega\|=n$, кратного $p-1$, число $s_{\omega}(\sigma)$ делится на $p$.

Рассмотрим построенную вьше систему $\alpha_{1}, \alpha_{2}, \ldots$ образуюших $\Omega_{U} \otimes \mathbb{Z}_{(p)}$. Для того чтобы доказать, что $\sigma \in \widetilde{\Lambda}_{p}(1) \otimes \mathbb{Z}_{(p)}$, необходимо доказать, что для любого не $p$-адического разбиения $\omega=\sum_{i} k_{i} \cdot(i)$, кратного $p-1$, коэффициент $r_{\omega}$ в разложении $(8)$ делится на $p$. Пусть $\omega$ - такое разбиение. Перепишем соотношение $(9)$ в виде

$$
s_{\omega}(\sigma)=r_{\omega} s_{\omega}\left(\alpha_{\omega}\right)+\sum_{\omega^{\prime} \supset \omega, \omega^{\prime} \neq \omega} r_{\omega^{\prime}} s_{\omega}\left(\alpha_{\omega^{\prime}}\right)
$$

По индукции для любого не $p$-адического, кратного $p-1$ разбиения $\omega^{\prime} \supset \omega, \omega^{\prime} \neq \omega$, $\left\|\omega^{\prime}\right\|=m(p-1)$, имеем, что $r_{\omega^{\prime}}$ делится на $p$. Если же в разбиении $\omega^{\prime}=\sum_{i} k_{i}^{\prime} \cdot(i)$ найдется $k_{i}^{\prime}=p^{k}-1$, то $s_{\omega}\left(\alpha_{\omega^{\prime}}\right)$ делится на $p$. Поэтому второе слагаемое в правой части (10) делится на $p$. По предположению $s_{\omega}(\sigma)$ также делится на $p$, а $s_{\omega}\left(\alpha_{\omega}\right)$ не делится на $p$, так как при нашем выборе $\omega$ имеем $\alpha_{\omega}=e \cdot \beta_{\omega}+\cdots$, где $e \in \mathbb{Z}_{(p)}-$ обратимьй элемент. Поэтому из $(10)$ следует, что $r_{\omega}$ делится на $p$. Теорема доказана.

СлЕдСтВИЕ 2. Элемент $\sigma \in \Omega_{U}$ является классом кобордизма многообразия $c$ действием $\mathbb{Z} / p$ с неподвижными многообразиями с тривиальными нормальными расслоениями тогда и только тогда, когда для всех разбиений $\omega$, кратных $p-1$, когомологические числа $s_{\omega}(\sigma),\|\omega\|=n$, делятся на $p$. 
СлЕДСТВИЕ 3. В каждом классе кобордизма многообразий $M^{n}, n \leqslant 4 p-6$, содержатся многообразия, несущие простое действие $\mathbb{Z} / p$.

В размерности $n=4 p-4$ уже имеется многообразие (например, $\mathbb{C P}^{2 p-2}$ ), в классе кобордизма которого не содержатся многообразия с простым действием $\mathbb{Z} / p$.

В работе Коннера и Флойда [1] методами, не используюшими технику формальных групп, показано, что класс кобордизма $\sigma \in \Omega_{U}$ содержит многообразие с сильно простым действием $\mathbb{Z} / p$ тогда и только тогда, когда все характеристические числа $s_{\omega}(\sigma)$ делятся на $p$. Точнее, множество классов кобордизма, содержащих многообразия с сильно простым действием $\mathbb{Z} / p$, совпадает с $\Omega_{U}$-модулем, натянутым на систему образуюших $Y^{0}=p, Y^{1}, Y^{2}, \ldots$, где $Y^{i} \in \Omega_{U}^{p^{i}-1}$ - так называемые многообразия Милнора, однозначно определяемые условиями: $s_{p_{-1}}\left(Y^{i}\right)=p, s_{\omega}\left(Y^{i}\right)$ делятся на $p$ при любом $\omega$. Если вместо $\Omega_{U}$-модулей рассматривать $\Omega_{U} \otimes \mathbb{Z}_{(p)}$-модули (этого вполне достаточно для описания множества классов кобордизма в терминах характеристических чисел), то в качестве представителей $Y^{i}$ можно взять элементы $\alpha_{p^{i}-1}^{(p)}$ из следствия 1 . Отсюда становится ясно, каким образом $\Omega_{U} \otimes \mathbb{Z}_{(p)}$-модуль $\Omega_{U}\left\langle p, Y^{1}, Y^{2}, \ldots\right\rangle \otimes \mathbb{Z}_{(p)}$, изучавшийся

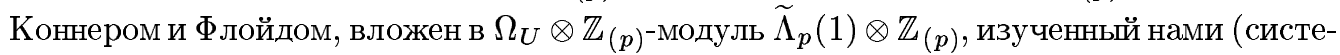
ма образуюших модуля Коннера и $\left.\widetilde{\Lambda}_{p}(1) \otimes \mathbb{Z}_{(p)}\right)$.

Заметим, наконец, что если некоторый класс $\sigma \in \Omega_{U}$ содержит представителя $M$, несущего сильно простое действие $\mathbb{Z} / p$, то необязательно любое простое действие $\mathbb{Z} / p$ на $M$ будет сильно простьм. Действительно, рассмотрим $M_{1}=\mathbb{C P}^{p-1}$ с действием образующей $\rho\left(z_{1}: \cdots: z_{p}\right)=\left(z_{1}: \rho z_{2}: \cdots: \rho^{p-1} z_{p}\right.$ ) (это действие с $p$ неподвижными точками является сильно простым действием) и $M_{2}=\mathbb{C P}^{1}, \rho\left(z_{1}: z_{2}\right)=\left(z_{1}: \rho z_{2}\right)$ (это простое действие с двумя неподвижньми точками не является сильно простьм). Тогда на $M=M_{1} \times M_{2}$ имеется два простых действия $\mathbb{Z} / p: \rho(a, b)=(\rho a, b)$ и $\rho(a, b)=(a, \rho b)$, $a \in \mathbb{C P}^{p-1}, b \in \mathbb{C P}^{1}$, первое из которых является сильно простым, а второе - нет.

Автор выражает глубокую благодарность научному руководителю д.ф.-м.н. В.М. Бухштаберу за полезные рекомендации и внимание к работе.

\section{СПИСОК ЦИТИРОВАННОЙ ЛИТЕРАТУРЫ}

[1] Коннер П., Флойд Э. Гладкие периодические отображения. М.: Мир, 1969.

[2] Новиков С. П. Методы алгебраической топологии с точки зрения теории кобордизмов // Изв. АН СССР. Сер. матем. 1967. Т. 31. С. 855-951.

[3] Бухштабер В. М., Новиков С. П. Формальные группы, степенные системы и операторы Адамса // Матем. сб. 1971. Т. 84(126). С. 81-118.

[4] Каспаров Г. Г. Инварианты классических линзовых многообразий в теории кобордизмов // Изв. АН СССР. Сер. матем. 1969. Т. 33. С. 735-747.

[5] Новиков С. П. Операторы Адамса и неподвижные точки // Изв. АН СССР. Сер. матем. 1968. T. 32. C. $1245-1263$.

[6] Бухштабер В. М. Характер Чженя-Дольда в теории кобордизмов. I // Матем. сб. 1970. T. $83(125)$. C. $575-595$.

[7] Стонг Р. Заметки по теории кобордизмов. М.: Мир, 1973.

Московский государственный университет им. М.В. Ломоносова E-mail: tpanov@nw.math.msu.su 